TITLE:

\title{
Magnetic force microscopy observation of antivortex core with perpendicular magnetization in patterned thin film of permalloy
}

\section{AUTHOR(S):}

Shigeto, K; Okuno, T; Mibu, K; Shinjo, T; Ono, T

\section{CITATION:}

Shigeto, K ... [et al]. Magnetic force microscopy observation of antivortex core with perpendicular magnetization in patterned thin film of permalloy. APPLIED PHYSICS LETTERS 2002, 80(22): 4190-4192

ISSUE DATE:

2002-06-03

URL:

http://hdl.handle.net/2433/50345

\section{RIGHT:}

Copyright 2002 American Institute of Physics. This article may be downloaded for personal use only. Any other use requires prior permission of the author and the American Institute of Physics. 


\title{
Magnetic force microscopy observation of antivortex core with perpendicular magnetization in patterned thin film of permalloy
}

\author{
K. Shigeto, ${ }^{\text {a) }}$ T. Okuno, K. Mibu, and T. Shinjo \\ Institute for Chemical Research, Kyoto University, Uji 611-0011, Japan \\ T. Ono \\ Graduate School of Engineering Science, Osaka University, Toyonaka 560-8531, Japan
}

(Received 14 December 2001; accepted for publication 17 April 2002)

\begin{abstract}
The cross-tie wall is a kind of magnetic domain wall composed of a main straight wall and crossing subwalls and observed in magnetic thin films. This wall contains two kinds of magnetic vortex structures: "circular vortex" and "antivortex." At the cores of both vortices, the existence of a spot with perpendicular magnetization has been theoretically predicted. We have detected the perpendicular magnetization spots at each vortex core and identified the direction of it by applying magnetic force microscopy imaging to cross-tie walls in patterned rectangular thin permalloy $\left(\mathrm{Ni}_{80} \mathrm{Fe}_{20}\right)$ films. We also fabricated magnetic structures that contain only antivortex by engineering the shape of thin films. (C) 2002 American Institute of Physics. [DOI: 10.1063/1.1483386]
\end{abstract}

The competition between exchange energy and magnetostatic energy provides us with various magnetic structures in ferromagnetic materials. ${ }^{1}$ The magnetic structure of crosstie walls observed in magnetic thin films is one of the most attractive structures, since it contains two types of magnetic vortex structures called "circular vortex" and "antivortex." Figure 1(a) shows the distribution of the magnetization $\mathbf{M}$ for the cross-tie wall in a rectangular permalloy $\left(\mathrm{Ni}_{80} \mathrm{Fe}_{20}\right)$ thin film $\left(450 \times 150 \mathrm{~nm}^{2}\right)$ obtained by micromagnetic simulation. The equilibrium magnetization is found by solving the dynamic Landau-Lifshitz-Gilbert equation. Only the magnetostatic energy and the exchange energy are taken into account. The square mesh size is $4.7 \times 4.7 \mathrm{~nm}^{2}$ and the thickness is set to be $5 \mathrm{~nm}$. The arrows corresponding to magnetization are displayed every three meshes. The cores of the circular vortex and the antivortex are marked $(+)$ and $(-)$, respectively. At both types of cores, the existence of a spot with perpendicular magnetization has been theoretically predicted. In the vicinity of the vortex core, the angle between adjacent spins would become increasingly larger if the spin directions were confined in-plane. Therefore, at the core of the vortex structure, the magnetization within a small region will turn out-of-plane not to increase the exchange energy. Transmission electron microscopy (TEM) images in Ref. 2 have shown that the cross-tie wall undoubtedly exists and the concept of such a magnetic vortex structure with a "turnedup" magnetization core has been introduced in a textbook. ${ }^{1}$ However, the conclusive experimental observations of the perpendicular component at the vortex cores has not been obtained as pointed out on p. 264 in the textbook of Ref. 1. Although the technique of TEM is well suited to the determination of the local direction and the magnitude of the inplane component of magnetization, ${ }^{3}$ it cannot detect the direction of perpendicular magnetization. It should be noted that the predicted size of the perpendicular magnetization

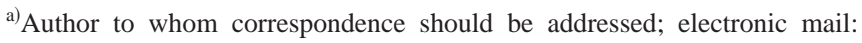
shigeto@ssc1.kuicr.kyoto-u.ac.jp
}

spot at the vortex core is fairly small, being severalnanometers scale in diameter. It is hard to distinguish a fraction of perpendicular magnetization from the surrounding inplane magnetization experimentally.

For the circular dots of permalloy, where only the circular vortex structure exists, we showed direct evidence of the perpendicular magnetization spot by magnetic force microscopy (MFM) imaging in a previous article. ${ }^{4}$ The MFM detects the magnetic force exerted on a magnetic probe tip when the tip is scanned over the surface of the sample. In the circular dots the in-plane magnetization rotates homogeneously and the magnetic charges appearing within the sensitivity of MFM originate only from the perpendicular component of magnetization. On the other hand, in the case of inhomogeneous magnetization distribution as in the cross-tie walls, the obtained MFM images tend to be more complicated, since the in-plane component also generates magnetic charges of $-\boldsymbol{\nabla} \cdot \mathbf{M}$, from which stray fields flow out. Although MFM observations of the cross-tie wall have been already reported by other groups,${ }^{5-8}$ the perpendicular magnetization core has not been reported yet. The success for the circular dots, ${ }^{4}$ however, informs us that the MFM has enough potential to detect the perpendicular component even in the case of the cross-tie walls. Figures 1(b) and 1(c) are the simulated MFM images based on the magnetic structure in Fig. 1(a). According to Saito et al., ${ }^{9}$ MFM images should be simulated by assuming that the tip interacts with the sample as a fixed point monopole. On the basis of this idea, the simulated MFM image is rendered from a contour plot of $d H_{z} / d z$ calculated at $40 \mathrm{~nm}$ above the simulated sample. Here, the direction of $z$ is defined to be perpendicular to the plane and $H_{z}$ is the $z$ component of magnetic field originating from the magnetic charges distributing on the sample surface. From the simulated MFM images, we can easily recognize the difference in the direction of the turned-up magnetization at the vortex cores in spite of the existence of strong signals originating from the in-plane magnetization components. The difference between the two images is caused only by the difference in the perpendicular magneti- 
(a)

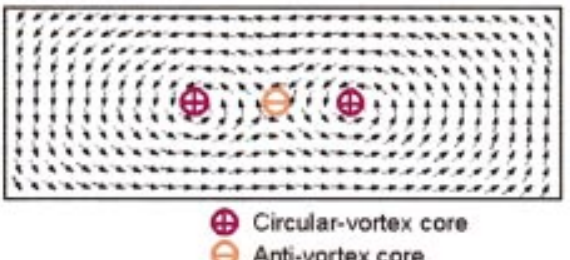

(b)
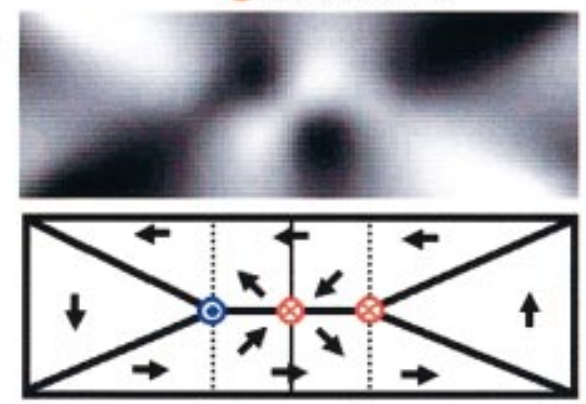

() Turned-up magnetization (2) Turned-down magnetization

(c)

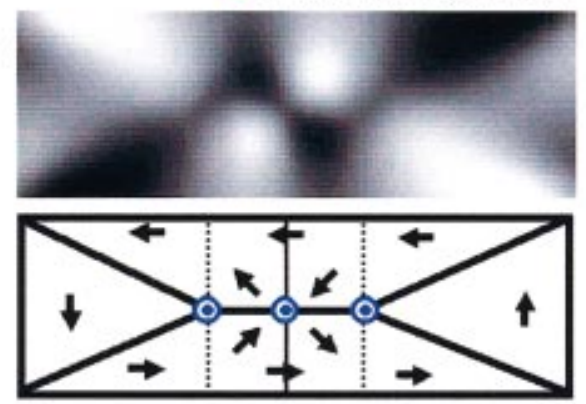

FIG. 1. (Color) (a) Result of micromagnetic simulation on the confined cross-tie wall in a rectangular permalloy dot $\left(450 \times 150 \mathrm{~nm}^{2}\right)$. Two circular vortices and one antivortex exist. (b),(c) Simulated MFM images with schematic illustrations of corresponding magnetization distributions.

zation direction as schematically shown in the figure.

In this paper, we report MFM measurements on the cross-tie walls confined in a patterned thin permalloy film and give clear evidence for the existence of the perpendicular magnetization cores for both circular vortex and antivortex structures. Furthermore, we successfully fabricated a special shape, where only the antivortex structure independently exists.

The samples were prepared by means of electron-beam lithography and deposition in an ultrahigh vacuum using an electron-beam gun. The desired patterns were prepared on thermally oxidized Si substrates, spin-coated with a layer of resist, and a layer of permalloy was subsequently deposited. Using a lift-off process, the resist was removed and permalloy patterns with designed shapes were left on the oxidized $\mathrm{Si}$ surface. The thickness of the prepared samples reported here is $50 \mathrm{~nm}$. In MFM observations, the instrument (Nanoscope IIIa: Digital Instruments) was operated in ac mode to detect the magnetic force acting between the cantilever tip and the permalloy patterns. Commercially available lowmoment ferromagnetic tips of CoCr (MESP-LM: Digital Instruments) was used in order to minimize the effect of stray fields from the tip. It should be noted that we do not always succeed in detecting the perpendicular component at the vortex core. It seems that a subtle difference in tip condition has influence on the resolution of MFM. With the condition where we get a magnetic contrast for a circular dot, we can Downloaded 04 Mar 2008 to 130.54 .110 .22 . Redistribution subject (a)

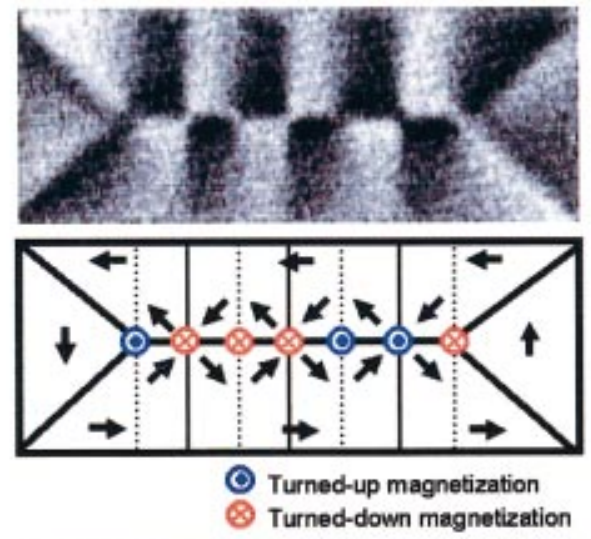

(b)
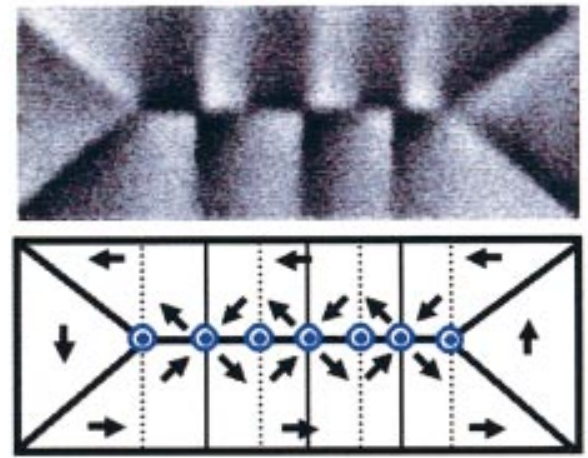

FIG. 2. (Color) Obtained MFM images of a rectangular permalloy dot (3 $\times 1 \mu \mathrm{m}^{2}$ ) with $50 \mathrm{~nm}$ in thickness: (a) an as-prepared state and (b) a remanent state after applying an external field of $1.5 \mathrm{~T}$ perpendicular (upward) to the plane. Schematic illustrations of realized magnetization distributions are also shown.

always detect the perpendicular component at the vortex core in the cross-tie wall. Circular dots, where the magnetic charges originate only from the perpendicular component at the vortex core, can be good standard samples to test the condition of MFM. The distance between the tip and the sample surface was set to $70 \mathrm{~nm}$ on the average. Sample scans were taken in air at ambient temperature.

Figures 2(a) and 2(b) show experimentally obtained MFM images of the cross-tie wall confined in a rectangular $\operatorname{dot}\left(3 \times 1 \mu \mathrm{m}^{2}\right)$. The MFM image in Fig. 2(a) was taken at an as-prepared state, while the image in Fig. 2(b) was taken at a remanent state after a magnetic field of $1.5 \mathrm{~T}$ was applied perpendicular (upward) to the plane. For both cases, we can easily recognize the core positions of four circular vortices and three antivortices. It is to be noted that in Fig. 2(b) only dark spots are recognized at the seven cores of the vortices, while in Fig. 2(a) four bright spots and three dark spots are observed at the cores. This is clear evidence that the bright and dark spots at the cores of the vortices correspond to the direction of the perpendicular magnetization component, since the direction at all cores aligns to that of the applied field for the image in Fig. 2(b). Schematic illustrations of the realized magnetization distribution are also shown in Figs. 2(a) and 2(b). Generally it has been believed that the perpendicular magnetization turns alternately to the opposite direction in order to decrease the magnetostatic energy. ${ }^{1,2,5}$ However, even in the case of the as-prepared state in Fig. 2(a), the directions are not periodic, suggesting that the interaction between cores is negligibly small.

As mentioned before, we have already known that the

license or copyright; see http://apl.aip.org/apl/copyright.jsp 
(a)
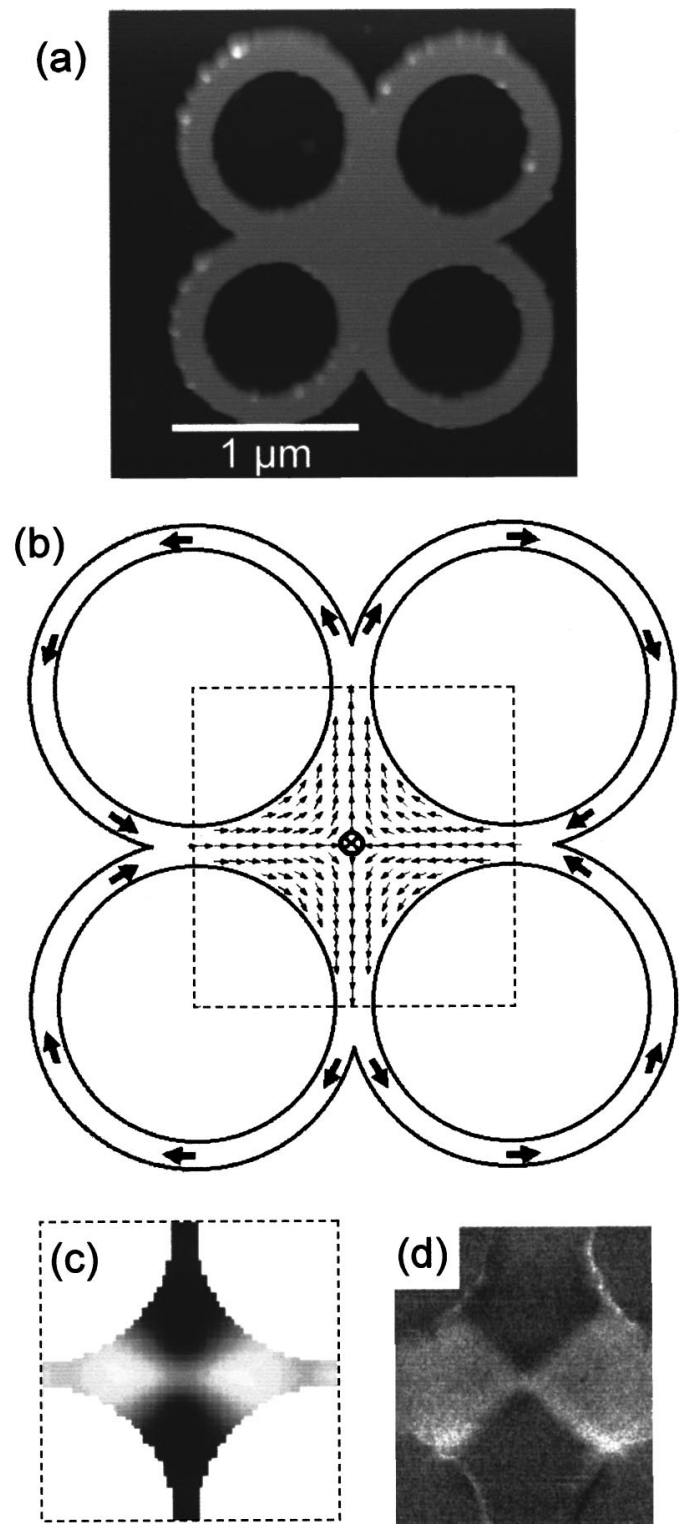

FIG. 3. (a) Topographic image of a specially designed sample having an asteroid in the middle by atomic force microscopy. (b) Result of micromagnetic simulation on the antivortex structure. (c) Simulated MFM image based on the magnetic structure shown in (b). (d) Obtained MFM image around the center of the sample at an as-prepared state.

isolated circular-vortex structure is realized in a circular dot. Similarly we tried to isolate the antivortex structure from the cross-tie wall using a specially designed sample having an asteroid in the middle. Figure 3(a) is a topographic image of the sample by atomic force microscopy. Figure 3(b) shows the magnetization distribution obtained by micromagnetic simulation, where the expected antivortex structure is reproduced. Figure 3(c) is a simulated MFM image based on the magnetic structure in Fig. 3(b) and it is assumed that the perpendicular magnetization at the core turns downward as shown in Fig. 3(b). It is characteristic that a bright signal appears at the core. A very similar image is actually obtained for an as-prepared sample [Fig. 3(d)]. When the perpendicular magnetization at the core turns upward, a dark signal should be observed at the core. In fact for some other samples with the same shape and size, dark signals were observed at the core. Therefore, it is certain that the expected magnetization distribution shown in Fig. 3(b) is realized in Fig. 3(d).

It has been known for a long time that two types of magnetic vortex structures, the circular vortex and the antivortex, exist in thin films as a part of the cross-tie wall, although we could not predict where the cross-tie wall is formed in a wide flat film. Engineering the shape of the film makes it possible not only to confine the cross-tie wall at the specific region but also to isolate the circular vortex and the antivortex from the cross-tie wall. Both vortex structures provide us nanoscale magnetic systems, i.e., the turned-up magnetization spots at the cores of the vortices. Nanoscale magnetic materials have boundaries, and the magnetic structures and the magnetization switching processes are generally influenced by the boundary conditions. In contrast, the nanoscale spots at the vortex cores spontaneously emerge in homogeneous material matrix and they do not feel the existence of material boundaries. Namely, the switching of the turned-up magnetization direction is not influenced by the material boundary of the sample itself. The visualized evidence that these turned-up spots undoubtedly exist convinces us of their importance again and shows us that the MFM is a powerful tool to investigate the switching property of the core magnetization. The switching processes of the core magnetization will be interesting issues in a future investigation.

The authors would like to thank Dr. Y. Suzuki for valuable discussions and Mr. Ishii for assistance in experiments. This work was supported by Grants-in-Aid for Research for the Future and Scientific Research (C) from the Japan Society for the Promotion of Science, and COE Research from the Ministry of Education, Culture, Sports, Science, and Technology.

${ }^{1}$ See, for example, A. Hubert and R. Schäfer, Magnetic Domains (Springer, Berlin, 1998)

${ }^{2}$ E. E. Huber, Jr., D. O. Smith, and J. B. Goodenough, J. Appl. Phys. 29, 294 (1958).

${ }^{3}$ R. Ploessl, J. N. Chapman, A. M. Thompson, J. Zweck, and H. Hoffmann, J. Appl. Phys. 73, 2447 (1993).

${ }^{4}$ T. Shinjo, T. Okuno, R. Hassdorf, K. Shigeto, and T. Ono, Science 289, 930 (2000).

${ }^{5}$ M. Löhndorf, A. Wadas, H. A. M. van den Berg, and R. Wiesendanger, Appl. Phys. Lett. 68, 3635 (1996).

${ }^{6}$ M. Schneider, St. Müller-Pfeiffer, and W. Zinn, J. Appl. Phys. 79, 8578 (1996).

${ }^{7}$ S. Huo, G. Pan, D. J. Mapps, W. W. Clegg, G. Heydon, W. M. Rainforth, H. A. Davies, J. E. L. Bishop, J. W. Tucker, and M. R. J. Gibbs, J. Appl. Phys. 87, 1096 (2000).

${ }^{8}$ S. T. Hung, Y. J. Tang, C. Y. Wong, S. K. Wong, and A. B. Pakhomov (unpublished)

${ }^{9}$ H. Saito, J. Chen, and S. Ishio, J. Magn. Magn. Mater. 191, 153 (1999). 